Chirurg 2019 $90: 287-292$

https://doi.org/10.1007/s00104-019-0936-y

Online publiziert: 15. März 2019

(c) Der/die Autor(en) 2019

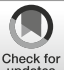

\section{H. J. Buhr ${ }^{1}$. C. Klinger ${ }^{1}$ K. S. Lehmann ${ }^{1}$ B. Strahwald ${ }^{2} \cdot$ A. Rieger ${ }^{2}$}

'Haus der Bundespressekonferenz, Deutsche Gesellschaft für Allgemein- und Viszeralchirurgie e. V., Berlin, Deutschland

${ }^{2}$ Institut für medizinische Informationsverarbeitung, Biometrie und Epidemiologie, LMU München, München, Deutschland

\title{
DGAV-Risikorechner für Eingriffe beim Kolon- und Rektumkarzinom
}

\section{Vorstellung eines chirurgischen Algorithmus zur Patientenpartizipation und Qualitätssicherung}

\section{Hintergrund}

Algorithmen erhalten zunehmend mehr Einfluss auf unseren Tagesablauf bzw. greifen, gewollt oder ungewollt, in Entscheidungen, Entscheidungswege und Prozesse unseres Lebens ein, wie z.B. das Finden der günstigsten Reiseverbindung oder die Kreditabfrage bei der SCHUFA. Künstliche Intelligenz, Big Data und entwickelte Algorithmen sind die modernen Schlagwörter unserer Zeit in der Tagespresse: „Meine Zeit steht in Rechnerzentren“, „Wie Google den Tod voraussagen will“, „Künstliche Intelligenz erstellt Prognosen für Klinikpatienten“ [8], „Der Todesalgorithmus: Computer berechnet Lebenserwartung " [10]. Das sind nur einige Schlagzeilen entsprechender Artikel in deutschen Zeitungen und Journalen. Alle dahinterstehenden Algorithmen beruhen auf umfangreichen Datensammlungen/-basen.

In der Tat werden weltweit umfangreiche Forschungen angestellt, sowohl von Google, Start-ups als auch von unabhängigen wissenschaftlichen Instituten, um auch im Gesundheitswesen Algorithmen $\mathrm{zu}$ entwickeln, die oft dem Ziel dienen, Kosten zu reduzieren bzw. einzusparen. So haben Mitarbeiter von Google Brain Daten zweier Kliniken in San Francisco und Chicago aus mehreren Jahren ausgewertet [11]. Hier wurden 216.000
Krankenhausaufenthalte von 114.000 verschiedenen Patienten analysiert. Der Datensatz betrug im Fazit nahezu 47 Mio. Einträge und ergab, dass die prognostizierte Sterbewahrscheinlichkeit genauer war als bei einem traditionell eingesetzten Vorhersagemodell.

Die ausgewählten Schlagzeilen und das genannte Beispiel lassen erahnen, was in Zukunft auf das Gesundheitswesen zukommen wird. Folgerichtig stellt sich die Frage, wie die Deutsche Gesellschaft für Allgemein- und Viszeralchirurgie (DGAV) aktiv in diese Entwicklung eingreifen kann bzw. soll oder ob kommerziellen Anbietern bzw. Außenstehenden dieses Feld überlassen wird.

\section{Material}

Die DGAV ist satzungsgemäß verpflichtet, für das Fach Allgemein- und Viszeralchirurgie Maßnahmen zur Qualitätssicherung und -verbesserung sowie für die Patientenpartizipation vorzunehmen. Hierzu war die Entwicklung von StuDoQ-Registern (Studien-, Dokumentations- und Qualitätszentrum der DGAV) neben der Einführung der Zertifizierungsverfahren ein wichtiger Schritt. Aktuell existieren zwölf webbasierte Register für allgemein- und viszeralchirurgische Eingriffe bzw. Me- thoden. In diesen wurden mehr als 150.000 Datensätze dokumentiert.

Allerdings dürfen diese Register keine „Datengräber“ darstellen. Daher verfolgt die DGAV - neben der wissenschaftlichen Analyse - das Ziel, den eingebenden chirurgischen Abteilungen einfach $\mathrm{zu}$ handhabende und zu erstellende Statistiken aus den Registern zur Verfügung zu stellen, um für die tägliche Arbeit in der Klinik und für die Qualitätssicherung Hilfestellungen zu geben und somit für den Patienten bzw. das Patientenwohlvon Nutzen zu sein.

Dies gilt insbesondere auch beim Abwägen eines Operationsverfahrens bei Patienten mit hohem Risikoprofil. Welche Komplikationen können mit welcher Wahrscheinlichkeit auftreten? Ist die erforderliche Intensivkapazität für einen Patienten mit hohem Risiko und für den geplanten Eingriff vorhanden? Wie beziehe ich den Patienten und seine Angehörigen in die Entscheidungsfindung ein, insbesondere um der berechtigten Forderung nach Patientenpartizipation nachzukommen? Der Patient muss über Operationsrisiken umfangreich und möglichst vollständig aufgeklärt sein.

Wenn in dem StuDoQ-Register nun 150.000 Datensätze vorhanden sind, ist es für die DGAV nur konsequent, diese auch für Antworten auf die gestellten 


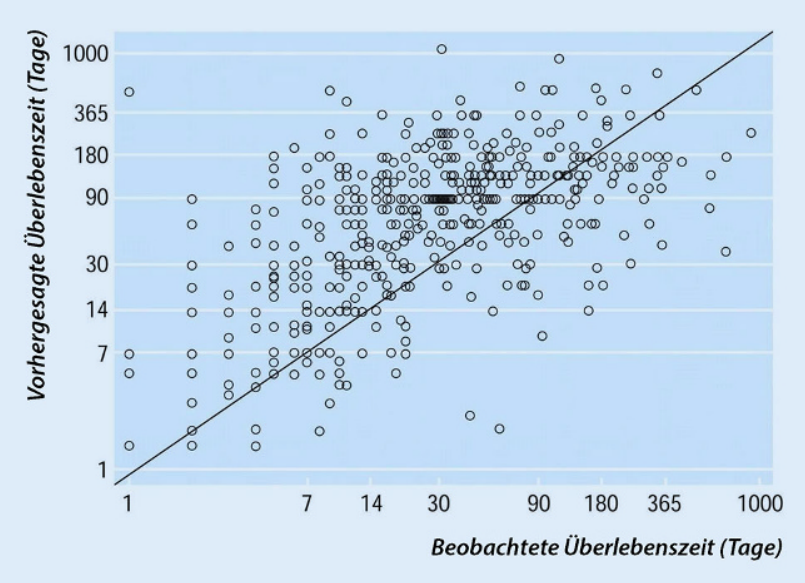

Abb. $1<$ Vorhergesagte und beobachtete Überlebenszeit bei $468 \mathrm{fi}$ nal erkrankten Patienten. Oberhalb der Diagonalen: überschätzt; unterhalb der Diagonalen: unterschätzt. (Adaptiert aus [12])

Fragen heranzuziehen, d. h. Algorithmen $\mathrm{zu}$ entwickeln, die anhand dieser Daten statistische Auskünfte über Risiken geben.

Ziel der DGAV ist es, je einen Risikorechner für das Kolonkarzinom und das Rektumkarzinom zu entwickeln.

\section{Risikoreduktion für intra- und postoperative Komplikationen}

Chirurgen sind es bei der klinischen Arbeit gewohnt, Risiken des Patienten prä-, intra- und postoperativ abzuwägen und diese bei der Wahl des Operationsverfahrens zu berücksichtigen bzw. mit allen Abwägungen in Entscheidungen einzubeziehen. Im Prinzip wird vor jedem Eingriff eine Kosten-Nutzen-Analyse aufgestellt, d. h., es wird versucht, die Frage $\mathrm{zu}$ beantworten, was dem Patienten in der Gesamtbetrachtung zuzumuten ist und welchen Nutzen er aus dem Eingriff für sich bzw. für seine postoperative Lebensqualität ziehen kann. Diese Abschätzung ist verständlicherweise stark vom Erfahrungsschatz des Chirurgen abhängig. Der Chirurg ist aber auch nicht frei davon, subjektive Gesichtspunkte mit in die Gesamtbeurteilung einfließen zu lassen. Und wie jeder aus eigener Erfahrung weiß, kommt es auch zu Fehleinschätzungen.

Dies belegt auch eine prospektive Kohortenstudie aus dem Jahr 2003 [4]: 343 Ärzte sollten bei 468 terminal erkrankten Patienten die Überlebenszeit einschätzen. Das Ergebnis dieser Beurteilung ist in Abb. 1 dargestellt: Die Diagonallinie präsentiert die genaue Vorhersage. Bei Patienten oberhalb der Linie wurde die Überlebenszeit über- und unterhalb der Linie unterschätzt. Die Autoren kommen zu dem Ergebnis, dass die Prognose in der Regel zu optimistisch eingeschätzt wurde, unabhängig vom Fach des Arztes und der Erkrankung.

Auf der anderen Seite beeinflusst auch die evidenzbasierte Studienlage die Entscheidungen der Ärzte. Sind sie bez. der Literatur auf der Höhe der Zeit und verfolgen Diskussionen auf Kongressen, werden die Ergebnisse entsprechender Studien immer ihre Berücksichtigung in der Risikobeurteilung finden. Dabei ist ihnen bewusst, dass dies alles nur Anhaltswerte sein können, statistisch errechnete Möglichkeiten, die auf die Patienten zutreffen können, aber nicht mit absoluter Sicherheit eintreten müssen. Es muss auch berücksichtigt werden, dass viele Studien Ergebnisse eines selektionierten Patientengutes widerspiegeln.

Persönliche Erfahrungen und evidenzbasiertes Medizinwissen fließen somit präoperativ permanent in die Entscheidungen ein: Was kann dem Patienten zugemutet werden? Welches Verfahren ist für ihn persönlich das richtige? Bringt der Eingriff das erwünschte Ergebnis? Beherrscht das Operationsteam (Ärzte wie Pflegepersonal) den Eingriff bzw. die eventuell postoperativ auftretenden Komplikationen? Profitiert der Patient wirklich von dem Eingriff Stichwort: krankheitsbezogene Lebensqualität?
Die Entscheidungsfindung muss auch ein wesentlicher Weiterbildungsschwerpunkt junger Chirurgen sein!

Weiterhin stellt sich natürlich auch die Frage: Wie sind der Patient und seine Angehörigen aufzuklären? Der Patient will und muss über das Verfahren, seine Risiken, Komplikationsmöglichkeiten genau aufgeklärt werden. Hierzu gilt es auch, gesetzliche Vorgaben des Bundesministeriums für Gesundheit (BMG) $\mathrm{zu}$ beachten [3].

\section{Der DGAV-Risikorechner}

Es ist sicher jedem bewusst, dass beide Faktoren, Erfahrung und Wissen, für eine Entscheidung Voraussetzung sind, aber dennoch nur vage Aussagen zulassen, wie auch die oben aufgeführte Studie belegt. Daher erscheint es sinnvoll, anhand der gesammelten StuDoQ-Registerdaten, insbesondere auch der dokumentierten Risikofaktoren der Patienten und der entsprechend aufgetretenen Komplikationen, Rechenmodelle/Algorithmen zu entwickeln, die es zulassen, statistische Wahrscheinlichkeiten zu berechnen, die das Risiko für Komplikationen beim einzelnen Patienten quantifizieren, d. h., das individuelle Risiko basierend auf einer Populationsberechnung zu schätzen. Dabei muss die statistische Unsicherheit jedem Anwender bewusst sein und auch dem Patienten ausreichend erläutert werden.

\section{Gründe für einen deutschen Risikorechner}

Weltweit sind diverse Risikorechner für unterschiedliche Zwecke bzw. Krankheitsbilder entwickelt worden. Einer der populärsten ist der Risk Calculator des American College of Surgeons (ACS; $[2,5,9])$, der auf den erfassten Daten des National Surgical Quality Improvement Program (NSQIP) beruht. In diese Datenbank fließen allerdings die Patientendaten mehrerer operativer Fächer (Gynäkologie, Urologie etc.) ein. Weiterhin ist die Zahl der erfassten Risikofaktoren und der Outcomeparameter deutlich begrenzt [2]. Die DGAV-Risikorechner beziehen sich ausschließlich auf die Registerdaten der beiden Karzinome 
des Dickdarms (Kolon und Rektum). Dies führt vermutlich $\mathrm{zu}$ einer besser belastbaren statistischen Vorhersage.

Publikationen [6] zeigen auch, dass Vorhersagen nicht mit einem Risikorechner eines anderen Landes oder Kontinents durchgeführt werden sollten: Anazawa et al. [1] haben für Daten japanischer Patienten die Ergebnisse des ACS-NSQIP-Risikorechners mit denen des japanischen National Clinic Data Base (NCD) - erstellt in Zusammenarbeit mit dem ACS - verglichen. Die Studie hatte zum Ziel, die Konsistenz und den Impact von Risikofaktoren für drei Eingriffe in Japan und USA (Hemikolektomie rechts, tiefe anteriore Rektumresektion und Pankreaskopfresektion) zu überprüfen. Das Ergebnis zeigte, dass lokale und nationale Risikomodelle in der Lage sind, die Mortalitätsrate vorherzusagen. Wurden aber die Modelle anderer Länder benutzt, war eine genaue Vorhersage nicht möglich. Sie kamen zu dem Fazit, dass nationale Risikomodelle essenziell für Qualitätsverbesserungen sind [1].

Degett et al. [7] kommen bei einer dänischen Untersuchung, in der ein englisches Risikomodell für die Mortalität (Association of Coloproctology of Great Britain and Ireland, ACPGBI) auf dänische Patienten mit kolorektalem Karzinom angewandt wurde, zum gleichen Schluss. Sie schreiben, dass geografische bzw. regionale/nationale Unterschiede bei der Organisation des Gesundheitssystems, der präoperativen Behandlung und Behandlungsqualität, in der allgemeinen Gesundheit der Bevölkerung wie z.B. hohe Raten an Rauchern, Komorbiditäten und sozioökonomischer Status zu dem festgestellten Unterschied führen und damit Risikomodelle anderer Länder nicht brauchbar sind.

\section{Ziel des DGAV-Risikorechners}

Der Risikorechner soll die Grundlage für die chirurgische Entscheidungsfindung (Erfahrung, Studienergebnisse) vor einem chirurgischen Eingriff beim Kolonund Rektumkarzinom mit statistischen Erkenntnissen erweitern bzw. ergänzen. Gleichzeitig besteht das Ziel, zusätzliche Informationen für das Aufklärungsge-

Chirurg 2019.90:287-292 https://doi.org/10.1007/s00104-019-0936-y

(c) Der/die Autor(en) 2019

H. J. Buhr · C. Klinger · K. S. Lehmann · B. Strahwald · A. Rieger

DGAV-Risikorechner für Eingriffe beim Kolon- und Rektumkarzinom. Vorstellung eines chirurgischen Algorithmus zur Patientenpartizipation und Qualitätssicherung

\section{Zusammenfassung}

Anhand großer Datensätze werden immer häufiger Algorithmen auch im Bereich der Gesundheit entwickelt, sei es zur Vorhersage von Behandlungsergebnissen oder der Lebenserwartung. In der Chirurgie wird es ebenfalls immer wichtiger, Komplikationen frühzeitig zu analysieren und zu senken. Ziel ist es, die Behandlungs- und Lebensqualität zu verbessern und somit das Patientenwohl zu steigern. Die Deutsche Gesellschaft für Allgemein- und Viszeralchirurgie (DGAV) hat zwölf StuDoQ-Register entwickelt, in denen pseudonymisierte Daten von insgesamt 150.000 Patienten erfasst sind. Mit den gesammelten Daten der Register StuDoQ|Kolonkarzinom und StuDoQ|Rektumkarzinom wurden am Institut für Medizinische Informationsverarbeitung,
Biometrie und Epidemiologie (IBE) der Ludwig-Maximilians-Universität München Risikomodelle entwickelt und validiert. Anhand der gesammelten Patientendaten ermittelt der Rechner die statistische Wahrscheinlichkeit des individuellen Komplikationsprofils des zu operierenden Patienten. Der Rechner stellt ein Hilfsmittel in der Entscheidungsfindung zur Operation dar. Der behandelnde Chirurg mit seiner Erfahrung bleibt der Letztverantwortliche für den Patienten.

Schlüsselwörter

Patientenpartizipation · Risikomodell . StuDoQ-Register · Behandlungsqualität . Lebensqualität

\section{German Society for General and Visceral Surgery (DGAV) risk calculator of interventions for colorectal cancer. Presentation of a surgical algorithm on patient participation and quality assurance}

\section{Abstract}

Algorithms are increasingly being developed on the basis of large data sets, also in the field of health, whether for predicting treatment outcomes or life-expectancy. In surgery it is also becoming increasingly more important to analyze complications at an early stage and to subsequently reduce them. The aim is to improve the quality of treatment and quality of life and thus to improve patient well-being. The German Society for General and Visceral Surgery (DGAV) has developed 12 StuDoQ registers in which pseudonymized data from a total of 150,000 patients are recorded. Risk models were developed and validated at the Institute for Medical Information Processing, Biometry and Epidemiology (IBE) of the
Ludwig Maximilian University in Munich using the collected data from the StuDoQ|colon cancer and StuDoQ|rectal cancer registers. Based on the collected patient data, the risk calculator determines the statistical probability of the individual complication profile of the patient who is to undergo surgery. The aim is to support surgeons and patients in the decision making process for the individual procedure. The surgeon with his individual experience ultimately remains responsible for the patient.

\section{Keywords}

Patient participation · Risk model · StuDoQ register · Treatment quality - Quality of life spräch mit dem Patienten und auch den Angehörigen zur Verfügung zu stellen.

Der Risikorechner hat zudem zwei weitere Vorteile: Er wurde auf der Basis der Daten deutscher Patienten entwickelt und diese Datenbasis stammt aus Häusern aller Versorgungsstufen mit unterschiedlichem Fallvolumen, also einem nicht über die Auswahl der Zentren selektionierten Krankengut.

Der Chirurg hat nun präoperativ die Möglichkeit, die Risikofaktoren des zu operierenden Patienten in den Rechner einzugeben und die Eintrittswahrscheinlichkeit für verschiedene Komplikationen bei dem vorgesehenen Operationsverfahren beim Kolon- und Rektumkar- 


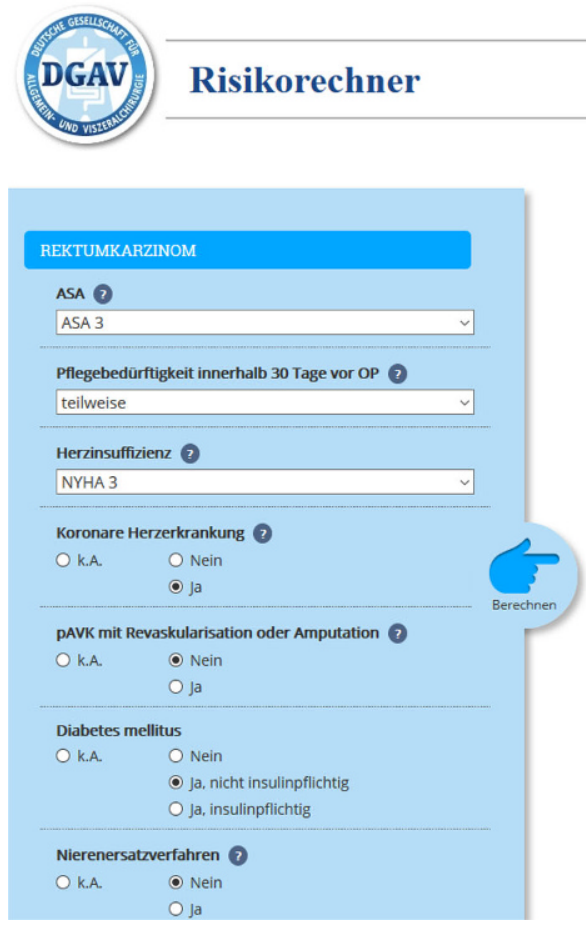

Risikorechner- Online Kurs - Abmelden

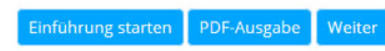

Abb. 2 ム Ergebnisansicht DGAV-Risikorechner. (Quelle: DGAV-Risikorechner)
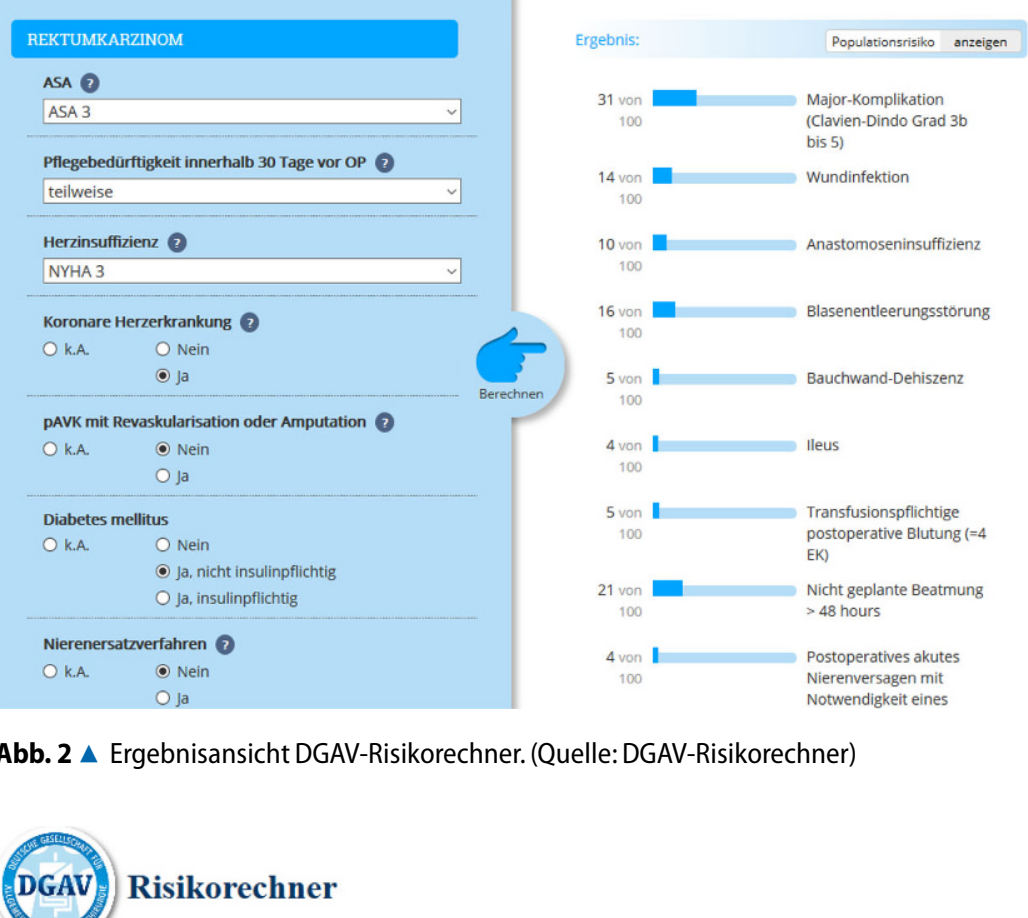

Risikorechner

\section{EINE KURZE EINFÜHRUNG IN 6 SCHRITTEN}

Bevor Sie den DGAV-Risikorechner erstmals nutzen, zeigen wir Ihnen die wichtigsten Grundlagen.

Dieser Online-Kurs ist vor der erstmaligen Nutzung des Risikorechners verpflichtend. Nach dem Abschluss werden Sie automatisch zum Risikorechner weitergeleitet.

ONLINE-KURS STARTEN

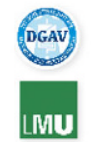

Der Online-Kurs wurde von der DGAV in Zusammenarbeit mit dem Institut für medizinische Informationsverarbeitung,
Biometrie und Epidemiologie der Biometrie und Epidemi
LMU Múnchen erstellt

Abb. 3 ^ Onlinekurs DGAV-Risikorechner. (Quelle: DGAV-Risikorechner)

zinom für seinen Patienten zu ermitteln und dementsprechend auch die Aufklärung des Patienten zu gestalten. Bei dem geplanten operativen Verfahren kann dann auf die Wahrscheinlichkeit einer Komplikation bei dem gewählten Operationsverfahren Bezug genommen werden, z.B. bei der Vorhaltung eines Intensivbettes oder bei der Auswahl der Operateure.
Der Chirurg kann mit dem Patienten und den Angehörigen ausführlich den Einfluss der einzelnen Risikofaktoren auf den Verlauf diskutieren und aufzeigen, welchen Einfluss eine Vorbehandlung wie z.B. eine optimale Diabeteseinstellung - erwarten lässt. In solchen Gesprächen bietet das statistisch errechnete Ergebnis und das geschätzte individuelle Komplikationsrisiko eine Hilfestellung, dem Patienten sein entsprechendes
Risikopotenzial darzustellen (• Abb. 2). Das Aufklärungsgespräch könnte somit informativer werden und die häufig in der Öffentlichkeit gestellte Forderung erfüllen, genauere und ausführlichere Informationen präoperativ zu übermitteln. Dies wäre zudem ein großer Beitrag zur Patientensicherheit.

Chirurg, Patient und Angehörige müssen sich bewusst sein bzw. es muss ihnen vermittelt werden, dass es sich um das geschätzte individuelle Risiko des betreffenden Patienten handelt, basierend auf der Populationsberechnung der in die zwei StuDoQ-Register eingegebenen pseudonymisierten Patientendaten. Die Beteiligten müssen wissen, dass eine statistische Unsicherheit bleibt und dass sich der individuelle Krankheitsverlauf auch anders entwickeln kann. Das heißt, eine Komplikation muss bei dem betreffenden Patienten nicht zwangsläufig eintreten oder ausbleiben, sondern der Wert bedeutet lediglich ein geschätztes individuelles Risiko, die statistische Möglichkeit einer Komplikation.

Diese Erkenntnis ist die Voraussetzung für den Gebrauch des DGAV-Risikorechners. Um dies jedem Benutzer bewusst zu machen, hat die DGAV einen E-Learning-Kurs entwickeln lassen, in dem in sechs Schritten die Entstehung des Rechners, die statistische Wahrscheinlichkeit und deren Einordnung und Bedeutung erläutert werden. Vor der ersten Benutzung des Risikorechners muss jeder Teilnehmer diesen Kurs online durchführen (• Abb. 3).

Daher rücken auch die Funktion, die Aufgaben und die Verpflichtung des behandelnden Chirurgen in den Mittelpunkt. Er trägt die Verantwortung und gemeinsam mit dem Patienten die Entscheidung zur Operation. Der Einsatz des DGAV-Risikorechners kann daher bei der endgültigen Bewertung eines Operationsverfahrens nur Hilfsmittel sein. Der erfahrene Chirurg ist weiterhin gefordert. Er hat für die Zusammenführung von Rechnerergebnis, eigener Erfahrung und Literatur zu sorgen.

Der Rechner soll jedem Chirurgen der Abteilung aber die Möglichkeit geben, den Eingriff mit den eventuellen Komplikationsrisiken abzuwägen und zu diskutieren. Er könnte für weiterzubilden- 


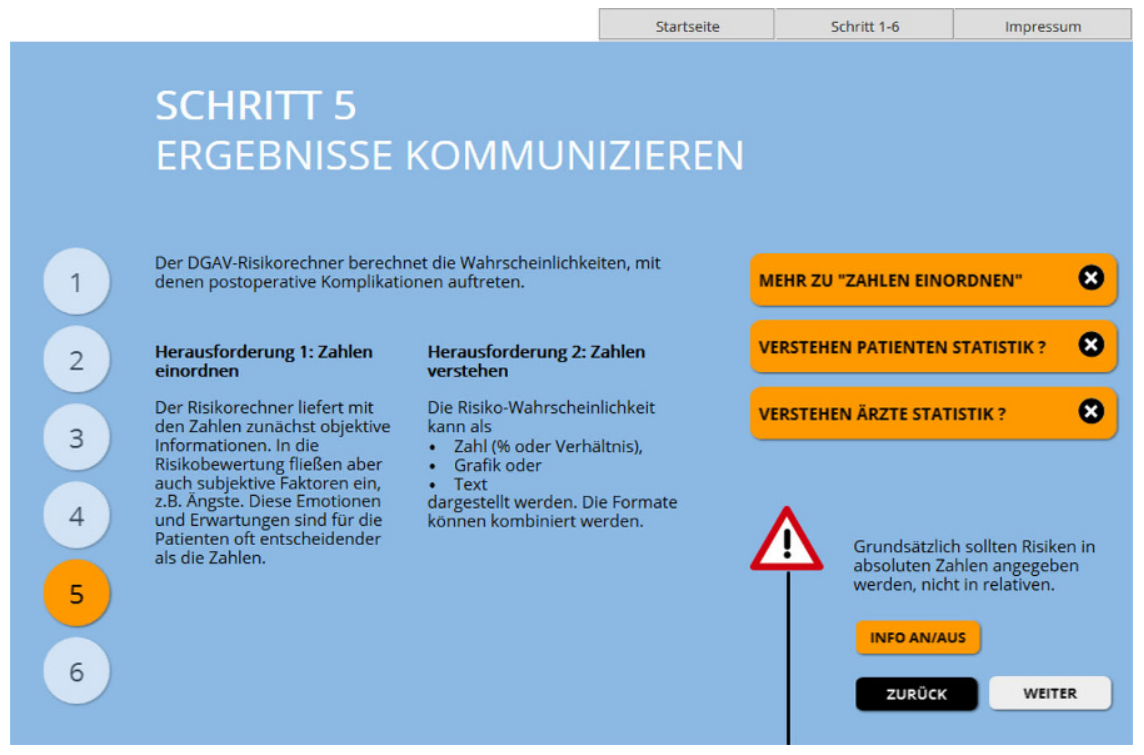

Abb. 4 ム Sechs Schritte des Vorbereitungskurses zur Benutzung des Risikorechners. (Quelle: DGAV-Risikorechner)

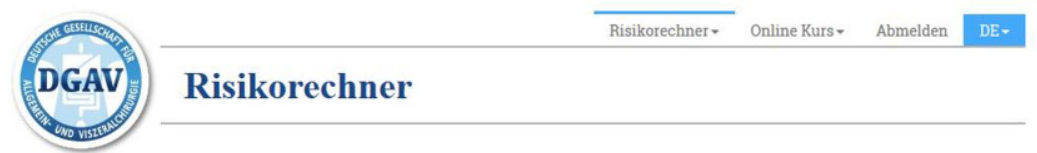

Bitte wählen Sie eine Diagnose!
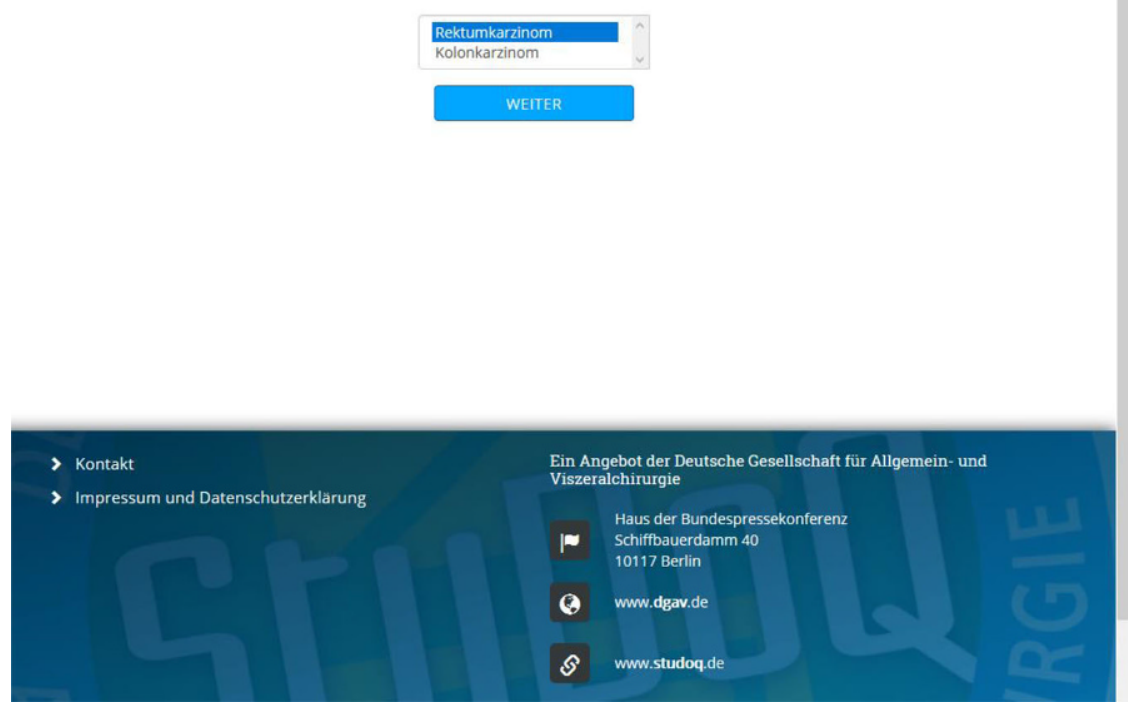

Abb. 5 ॥ Organauswahl im Risikorechner. (Quelle: DGAV Risikorechner)

de Chirurgen ein wichtiges Instrument für die zukünftige Entscheidungsfindung und für die Entwicklung eigener Erfahrungen werden.

Letztendlich stellen sich vier entscheidende Fragen. Erstens: Wird die Voraussage einer möglichen Komplikation durch den Rechner besser? Zweitens: Wirkt sich die Anwendung des Rechners

\section{Entwicklung des DGAV- Risikorechners}

Auf der Basis der in den DGAV-Registern StuDoQ|Kolonkarzinom und StuDoQ|Rektumkarzinom erfassten Daten wurde ein Rechenmodell für die Risikorechner für die kolorektalen Karzinome entwickelt. Es flossen 30 Risikofaktoren, 15 intra- und postoperative Komplikationen bzw. Outcomeparameter - wie Majorkomplikationen, Anastomoseninsuffizienzen, tiefe chirurgische Wundinfekte - sowie das durchgeführte Operationsverfahren ein.

Die Entwicklung und Validierung des statistischen Modells wurde ausführlich von Crispin et al. [6] beschrieben. Die Validierung in dieser Publikation bezog sich auf die Daten von 6729 Kolon- und 4381 Rektumkarzinompatienten.

Das statistische Modell wurde in die webbasierten DGAV-Risikorechner übertragen. Die technische Umsetzung erlaubt die Ergänzung um Modelle für weitere Erkrankungen bzw. Behandlungen.

\section{Anwendung des DGAV- Risikorechners}

Der DGAV-Risikorechner ist zunächst nur mit einem Benutzernamen und Passwort zugänglich. Die Anmeldung erfordert zudem jedes Mal die Bestätigung, dass der Disclaimer gelesen und dem Inhalt zugestimmt wird. Jeder Nutzer wird bei der erstmaligen Anmeldung zu dem bereits erwähnten Vorbereitungskurs mit seinen sechs Schritten geleitet (- Abb. 4). Erst nach dessen Abschluss kann auf den eigentlichen Risikorechner zugegriffen werden. Wird der Vorbereitungskurs beim ersten Besuch nicht abgeschlossen, erfolgt bei jedem weiteren Besuch erneut die Weiterleitung zum Kurs.

Im Risikorechner erfolgt im ersten Schritt immer die Auswahl des Tumorleidens (aktuell Rektum- oder Kolonkarzinom, - Abb. 5). Im zweiten Schritt erfolgt die Eingabe des geplanten Operationsverfahrens und aller Risikofaktoren des betreffenden Patienten. AnschlieBend wird das Komplikationsmuster mit 
den entsprechenden statistischen Wahrscheinlichkeiten errechnet.

Mit dem Rechner kann dem Patienten auch demonstriert werden, welchen Einfluss eliminierte Risikofaktoren auf das Komplikationsprofil haben. Dies könnte dazu führen, dass der Patient präoperativ besser auf die Operation vorbereitet wird.

Die Ergebnisse des Rechners können als PDF-Datei exportiert und der Krankenakte zugefügt werden.

\section{Korrespondenzadresse}

\section{Prof. Dr. H. J. Buhr}

Haus der Bundespressekonferenz,

Deutsche Gesellschaft für Allgemein- und

Viszeralchirurgie e.V.

Schiffbauerdamm 40, 10117 Berlin,

Deutschland

hbuhr@dgav.de

\section{Einhaltung ethischer Richtlinien}

Interessenkonflikt. H.J. Buhr, C. Klinger, K.S. Lehmann, B. Strahwald und A. Rieger geben an, dass kein Interessenkonflikt besteht.

Dieser Beitrag beinhaltet keine von den Autoren durchgeführten Studien an Menschen oder Tieren.

Open Access. Dieser Artikel wird unter der Creative Commons Namensnennung 4.0 International Lizenz (http://creativecommons.org/licenses/by/4.0/deed. de) veröffentlicht, welche die Nutzung, Vervielfältigung, Bearbeitung, Verbreitung und Wiedergabe in jeglichem Medium und Format erlaubt, sofern Sie den/die ursprünglichen Autor(en) und die Quelle ordnungsgemäßnennen, einen Link zur Creative Commons Lizenz beifügen und angeben, ob Änderungen vorgenommen wurden.

\section{Literatur}

1. Anazawa T, Paruch JL, Miyata H et al (2015) Comparison of national operative mortality in gastroenterological surgery using web-based prospective data entry systems. Medicine (Baltimore) 94:e2194. https://doi.org/10.1097/MD. 0000000000002194

2. Bilimoria KY, Liu Y, Paruch JL et al (2013) Development and evaluation of the universal ACS NSQIP surgical risk calculator: a decision aid and informed consent tool for patients and surgeons. J Am Coll Surg 217:833-842.e1-3. https://doi.org/ 10.1016/j.jamcollsurg.2013.07.385

3. Bundesgesundheitsministerium (Hrsg) BMG Patientenrechtegesetz. https://www. bundesgesundheitsministerium.de/service/ begriffe-von-a-z/p/patientenrechtegesetz.html. Zugegriffen:21.Dez. 2018
4. Christakis NA, Lamont EB (2000) Extent and determinants of error in physicians' prognoses in terminally ill patients: prospective cohort study. West J Med 172:310-313

5. Cohen ME, Liu Y, Ko CY, Hall BL (2017) An examination of American College of Surgeons NSQIP surgical risk calculator accuracy. J Am Coll Surg 224:787-795.e1. https://doi.org/10.1016/j. jamcollsurg.2016.12.057

6. Crispin A, Klinger C, Rieger A et al (2017) The DGAV risk calculator: development and validation of statistical models for a web-based instrument predicting complications of colorectal cancer surgery. Int J Colorectal Dis. https://doi.org/10. 1007/s00384-017-2869-6

7. Degett TH, Roikjær O, Iversen LH, Gögenur I (2018) A model predicting operative mortality in the UK has only limited value in Denmark. Int J Colorectal Dis 33:141-147. https://doi.org/10.1007/s00384017-2937-y

8. KMA Forschung Künstliche Intelligenz von Google erstellt Prognosen für Klinik-Patienten. https:// www.kma-online.de/aktuelles/it-digital-health/ detail/kuenstliche-intelligenz-von-googleerstellt-prognosen-fuer-klinik-patienten-a37894.Zugegriffen:21.Dez. 2018

9. Lubitz AL, Chan E, Zarif D et al (2017) American college of surgeons NSQIP risk calculator accuracy for emergent and elective colorectal operations. J Am Coll Surg 225:601-611. https://doi.org/10 1016/j.jamcollsurg.2017.07.1069

10. NDR Der Todesalgorithmus: Computer berechnet Lebenserwartung. https://daserste.ndr.de/ panorama/archiv/2017/Der-TodesalgorithmusComputer-berechnet-Lebenserwartung, todesalgorithmus112.html. Zugegriffen: 21. Dez. 2018

11. Rajkomar A, Oren E, Chen K et al (2018) Scalable and accurate deep learning with electronic health records. Npj Digit Med 1:18. https://doi.org/10. 1038/s41746-018-0029-1

12. Christakis NA, Lamont EB (2000) Extent and determinants of error in doctors' prognoses in terminally ill patients: prospective cohort study. BMJ320(7233):469-472
Hier steht eine Anzeige. Springer 